Proyecciones Journal of Mathematics

Vol. 40, No 6, pp. 1507-1519, December 2021.

Universidad Católica del Norte

Antofagasta - Chile

\title{
P-adic discrete semigroup of contractions
}

Abdelkhalek El amrani

Sidi Mohamed Ben Abdellah University, Morocco

Jawad Ettayb

Sidi Mohamed Ben Abdellah University, Morocco

and

Aziz Blali

Sidi Mohamed Ben Abdellah University, Morocco

Received: August 2020. Accepted: June 2021

\begin{abstract}
Let $A \in B(X)$ be a spectral operator on a non-archimedean Banach space over $\mathbf{C}_{p}$. In this paper, we give a necessary and sufficient condition on the resolvent of $A$ so that the discrete semigroup consisting of powers of $A$ is contractions.
\end{abstract}

Subjclass [2010]: 11E95, 47A10, 47D03.

Keywords: Non-archimedean Banach spaces, spectral operator, discrete semigroup of contractions. 


\section{Introduction and Preliminaries}

In the archimedean operators theory, necessary and sufficient conditions on the resolvent of a densely defined closed linear operator for it to be the infinitesimal generator of a strongly continuous semigroup $(T(s))_{s \in \mathbf{R}^{+}}$ such that there is $M \geq 1,\|T(s)\| \leq M$. In particular, we have the following theorem and its corollary.

Theorem 1.1. [7] A necessary and sufficient condition that a closed linear operator $A$ with dense domain be the infinitesimal generator of a strongly continuous semigroup $(T(s))_{s \in \Omega_{r}}$ such that for all $s \in \mathbf{R}^{+},\|T(s)\| \leq M$ is that

$$
\left\|R_{\lambda}(A)^{n}\right\| \leq \frac{M}{\lambda^{n}}
$$

for $\lambda>0$ and $n \in \mathbf{N}$, where $R_{\lambda}(A)=(\lambda I-A)^{-1}$.

Corollary 1.2. [7] A necessary and sufficient condition that a closed linear operator $A$ with dense domain be the infinitesimal generator of a strongly continuous semigroup $(T(s))_{s \in \Omega_{r}}$ such that for all $s \in \mathbf{R}^{+},\|T(s)\| \leq 1$ is that

$$
\left\|R_{\lambda}(A)\right\| \leq \frac{M}{\lambda}
$$

for $\lambda>0$.

Throughout this paper, $X$ is a non-archimedean (n.a) Banach space over a (n.a) non trivially complete valued field $\mathbf{K}$ which is also algebraically closed with valuation $|\cdot|, B(X)$ denote the set of all bounded linear operators on $X$ into $X, \mathbf{Q}_{p}$ is the field of p-adic numbers ( $p \geq 2$ being a prime) equipped with $\mathrm{p}$-adic valuation $|\cdot|_{p}, \mathbf{Z}_{p}$ denotes the ring of $p$-adic integers of $\mathbf{Z}_{p}$ is the unit ball of $\mathbf{Q}_{p}$. For more details and related issues, we refer to [4] and [6]. We denote the completion of algebraic closure of $\mathbf{Q}_{p}$ under the p-adic abolute value $|\cdot|_{p}$ by $\mathbf{C}_{p}$ (see [4]). Let $r>0, \Omega_{r}$ be the clopen ball of $\mathbf{K}$ centred at 0 with radius $r>0$, that is $\Omega_{r}=\{t \in \mathbf{K}:|t|<r\}$. Recall that a free non-archimedean Banach space $X$ is a non-archimedean Banach space for which there exists a family $\left(e_{i}\right)_{i \in I}$ in $X \backslash\{0\}$ such that any element $x \in X$ can be written in the form of a convergent sum $x=\sum_{i \in I} x_{i} e_{i}$, $x_{i} \in \mathbf{K}$, i.e., $\lim _{i \in I} x_{i} e_{i}=0$ (the limit is with respect to the Fréchet filter on $I)$ and $\|x\|=\sup _{i \in I}\left|x_{i}\right|\left\|e_{i}\right\|$. Let $X$ be a free non-archimedean Banach 
space, recall that every bounded linear operator $A$ on $X$ can be written in a unique fashion as a pointwise convergent series, that is, there exists an infinite matrix $\left(a_{i, j}\right)_{(i, j) \in \mathbf{N} \times \mathbf{N}}$ with coefficients in $\mathbf{K}$ such that

$$
A=\sum_{i, j \in \mathbf{N}} a_{i, j} e_{j}^{\prime} \otimes e_{i}, \text { and } \forall j \in \mathbf{N}, \quad \lim _{i \rightarrow \infty}\left|a_{i, j}\right|\left\|e_{i}\right\|=0,
$$

where $(\forall i \geq 1) e_{i}^{\prime}(u)=u_{i}\left(e_{i}^{\prime}\right.$ is the linear form associated with $\left.e_{i}\right)$.

Moreover, for each $j \in \mathbf{N}, \quad \mathrm{Ae}_{j}=\sum_{i \in \mathbf{N}} a_{i j} e_{i}$ and its norm is defined by

$$
\|A\|=\sup _{i, j} \frac{\left|a_{i j}\right|\left\|e_{i}\right\|}{\left\|e_{j}\right\|} \text {. }
$$

For more details see [1], Proposition 3.7.

Definition 1.3. [1] Let $\omega=\left(\omega_{i}\right)_{i}$ be a sequence of non-zero elements of K. We define $\mathbf{E}_{\omega}$ by

$\mathbf{E}_{\omega}=\left\{x=\left(x_{i}\right)_{i}: \forall i \in \mathbf{N}, x_{i} \in \mathbf{K}\right.$, and $\left.\lim _{i \rightarrow \infty}\left|\omega_{i}\right|^{\frac{1}{2}}\left|x_{i}\right|=0\right\}$,

it is equipped with the norm

$\forall x \in \mathbf{E}_{\omega}: x=\left(x_{i}\right)_{i},\|x\|=\sup _{i \in \mathbf{N}}\left(\left|\omega_{i}\right|^{\frac{1}{2}}\left|x_{i}\right|\right)$.

Remark 1.4. (1) [1], Exemple 2.21. The space $\left(\mathbf{E}_{\omega},\|\cdot\|\right)$ is a non archimedean Banach space.

(2) If

$$
\begin{aligned}
\langle\cdot, \cdot\rangle: \mathbf{E}_{\omega} \times \mathbf{E}_{\omega} & \longrightarrow \mathbf{K} \\
(x, y) & \mapsto \sum_{i=0}^{\infty} x_{i} y_{i} \omega_{i}
\end{aligned}
$$

where $x=\left(x_{i}\right)_{i}$ and $y=\left(y_{i}\right)_{i}$. Then, the space $\left(\mathbf{E}_{\omega},\|\cdot\|,\langle\cdot, \cdot\rangle\right)$ is called a p-adic (or non archimedean) Hilbert space.

(2) The orthogonal basis $\left\{e_{i}, i \in \mathbf{N}\right\}$ is called the canonical basis of $\mathbf{E}_{\omega}$, where for all $i \in \mathbf{N},\left\|e_{i}\right\|=\left|\omega_{i}\right|^{\frac{1}{2}}$. 
Definition 1.5. [6] Let $A \in B(X)$, set $\nu(A)=\inf _{n}\left\|A^{n}\right\|^{\frac{1}{n}}=\lim _{n}\left\|A^{n}\right\|^{\frac{1}{n}}$, $A$ is said to be a spectral operator if $\sup \left\{|\lambda|: \lambda^{n} \in \sigma(A)\right\}={ }^{n}(A)$. For $A \in B(X)$, set $U_{A}=\left\{\lambda \in \mathbf{K}:(I-\lambda A)^{-1}\right.$ exists in $\left.B(X)\right\}$. ( $U_{A}$ is open and $\left.0 \in U_{A}\right)$ and

$$
C_{A}=\left\{\alpha \in \mathbf{K}: B(0,|\beta|) \subset U_{A} \text { for some } \beta \in \mathbf{K},|\beta|>|\alpha|\right\} .
$$

Proposition 1.6. [6] Let $A \in B(X)$, the following are equivalent.

(i) $A$ is a spectral operator.

(ii) For all $\lambda \in C_{A},(I-\lambda A)^{-1}=\sum_{n=0}^{\infty} \lambda^{n} A^{n}$.

(iii) For each $\alpha \in C_{A}^{*}$, the function $\lambda \mapsto(I-\lambda A)^{-1}$ is analytic on $B(0,|\alpha|)$.

\section{Discrete semigroups of bounded linear operators on non- archimedean Banach space}

We begin with the following definition.

Definition 2.1. Let $X$ be a non-archimedean Banach space over K. A family $(T(n))_{n \in \mathbf{N}}$ of bounded linear operators from $X$ into $X$ is said to be a discrete semigroup of bounded linear operators on $X$ if

(i) $T(0)=I$, where $I$ is the unit operator of $X$;

(ii) For all $m, n \in \mathbf{N}, T(m+n)=T(m) T(n)$.

Remark 2.2. Let $A \in B(X), T(n)=A^{n}$ is a discrete semigroup of bounded linear operators on $X$, its generator $A$.

Definition 2.3. Let $X$ be a non-archimedean Banach space over $\mathbf{K}$. A discrete semigroup $(T(n))_{n \in \mathbf{N}}$ is said to be uniformly bounded if $\sup _{n \in \mathbf{N}}\|T(n)\|$ is finite.

In contrast with the classical setting, we have the following example. 
Example 2.4. Let $\mathbf{K}=\mathbf{Q}_{p}$, if

$$
A=\left(\begin{array}{ll}
1 & 1 \\
0 & 1
\end{array}\right)
$$

then $A$ generate a discrete semigroup of bounded linear operators $(T(n))_{n \in \mathbf{N}}$ given by:

$$
\forall n \in \mathbf{N}, T(n)=\left(\begin{array}{cc}
1 & n \\
0 & 1
\end{array}\right) .
$$

In fact, it is easy to check that:

(i) $T(0)=I$ where $I$ is the unit operator on $\mathbf{Q}_{p}^{2}$.

(ii) For all $m, n \in \mathbf{N}, T(m+n)=T(m) T(n)$.

(iii) For all $z=(x, y) \in \mathbf{Q}_{p}^{2}, n \in \mathbf{N}$, we have

$$
\begin{aligned}
\|T(n) z\| & =\left\|\left(\begin{array}{c}
x+n y \\
y
\end{array}\right)\right\|, \\
& =\max \left\{|x+n y|_{p},|y|_{p}\right\}, \\
& \leq \max \left\{|x|_{p},|n y|_{p},|y|_{p}\right\}, \\
& \leq \max \left\{|x|_{p},|y|\right\} \text { with }|n|_{p} \leq 1, \\
& \leq\|z\| .
\end{aligned}
$$

Then $(T(n))_{n \in \mathbf{N}}$ is an uniformly bounded discrete semigroup of bounded linear operators on $\mathbf{Q}_{p}^{2}$.

We have the following definition.

Definition 2.5. Let $X$ be a non-archimedean Banach space over $\mathbf{K}$, let $(T(n))_{n \in \mathbf{N}}$ be a discrete semigroup of bounded linear operators on $X$, $(T(n))_{n \in \mathbf{N}}$ is said to be semigroup of contractions if for all $n \in \mathbf{N},\|T(n)\| \leq$ 1.

Example 2.6. Let $X=\mathbf{E}_{\omega}$ where for all $i \in \mathbf{N}, \omega_{i}=p^{i}$. Let $A$ be unilateral shift given by

for all $i \in \mathbf{N}, A e_{i}=e_{i+1}$.

Then, for all $n \in \mathbf{N}, A^{n} e_{i}=e_{n+i}$, hence, for all $n \in \mathbf{N}, \frac{\left\|A^{n} e_{i}\right\|}{\left\|e_{i}\right\|}=p^{\frac{-n}{2}} \leq$ 1. Consequently, for all $n \in \mathbf{N},\left\|A^{n}\right\| \leq 1$. Moreover, $\left(A^{n}\right)_{n \in \mathbf{N}}$ is a discrete semigroup of contractions on $\mathbf{E}_{\omega}$. 
We start with the following statements.

Lemma 2.7. Let $(T(n))_{n \in \mathbf{N}}$ be a discrete semigroup of bounded linear operators on $X$ such that $\sup \|T(n)\| \leq M$. Then there exists an equivalent norm on $X$ such that $(T(n))_{n \in \mathbf{N}}$ becomes a contraction.

Proof. Set: $|x|_{1}=\sup _{n \in \mathbf{N}}\|T(n) x\|$. We have $\|T(0)\|=1$ and $(\forall n \in \mathbf{N}),\|T(n)\| \leq M$, then $(\forall x \in X)\|x\| \leq|x|_{1} \leq M\|x\|^{\cdot}$ Hence $|\cdot|_{1}$ is a norm on $X$ which is equivalent to the original norm $\|\cdot\|$ on $X$ Furthermore, for all $x \in X$, all $n \in \mathbf{N}, \quad|T(n) x|_{1}=\sup _{m \in \mathbf{N}}\|T(n) T(m) x\|=\sup _{m \in \mathbf{N}} \| T(n+$ $m) x\left\|=\sup _{m \geq n}\right\| T(m) x\left\|\leq \sup _{m \in \mathbf{N}}\right\| T(m) x \|=|x|_{1}$.

In the next Proposition, we assume that $\mathbf{Q}_{p} \subset \mathbf{K}$.

Proposition 2.8. The set of all discrete semigroup of contractions form a $\mathbf{Z}_{p}$-subspace of $B(X)$.

Proof. Set $C$ denote the set of all discrete semigroup of contractions on $X$ into $X$.

(1) $\left\|I_{X}\right\| \leq 1$, Hence $C \neq \emptyset$.

(2) Let $(T(n))_{n \in \mathbf{N}}$ and $(S(n))_{n \in \mathbf{N}}$ in $C$ and $\lambda \in \mathbf{Z}_{p}$, we have

$$
\begin{aligned}
\|T(n)+\lambda S(n)\| & \leq \max \{\|T(n)\| ;|\lambda| \| S(n) \mid\} \\
& \leq 1
\end{aligned}
$$

Hence, for all $n \in \mathbf{N}$ and for all $\lambda \in \mathbf{Z}_{p}, T(n)+\lambda S(n) \in C$.

In the rest of this paper, for $A \in B(X)$ be a spectral operator such that $\sup _{\|}\left\|A^{n}\right\|$ is finite, we assume that $U_{A}=B(0,1)$ where $\mathrm{B}(0,1)=\{\lambda \in \mathbf{K}$ : $n \in \mathbf{N}$

$|\lambda|<1\}$, and for all $\lambda \in U_{A}, R(\lambda, A)=(I-\lambda A)^{-1}$.

Proposition 2.9. Let $X$ be a non-archimedean Banach space over $\mathbf{K}$, let $A$ be a spectral operator and there is $M \geq 1$ such that $\sup _{n \in \mathbf{N}}\left\|A^{n}\right\| \leq M$. Then,

for all $\lambda \in C_{A},\|R(\lambda, A)\| \leq M$. 
Proof. By Proposition 1.6, for all $\lambda \in C_{A}, \lim _{n \rightarrow \infty}|\lambda|^{n}\left\|A^{n}\right\|=0$, hence

$$
\begin{aligned}
\|R(\lambda, T)\| & =\left\|\sum_{n=0}^{\infty} \lambda^{n} A^{n}\right\| \\
& \leq M \max _{n \in \mathbf{N}}\left|\lambda^{n}\right| \\
& =M .
\end{aligned}
$$

Proposition 2.10. Let $A \in B(X)$ be a spectral operator, let $\left(A^{n}\right)_{n \in \mathbf{N}}$ be a discrete semigroup of bounded linear operators on $X$ such that $\sup _{n \in \mathbf{N}}\left\|A^{n}\right\|$ is finite and $U_{A}=B(0,1)$. Then, for all $\lambda, \mu \in C_{A}$,

$$
\lambda R(\lambda, A)-\mu R(\mu, A)=(\lambda-\mu) R(\lambda, A) R(\mu, A) .
$$

Proof. Let $\lambda, \mu \in C_{A}$, we have

$$
\begin{aligned}
& \lambda R(\lambda, A)(I-\mu A) R(\mu, A)-\mu R(\lambda, A)(I-\lambda A) R(\mu, A) \\
(2.1)= & \lambda R(\lambda, A) R(\mu, A)-\lambda \mu R(\lambda, A) A R(\mu, A)-\mu R(\lambda, A) R(\mu, A) \\
+ & \lambda \mu R(\lambda, A) A R(\mu, A) ; \\
= & \lambda R(\lambda, A) R(\mu, A)-\mu R(\lambda, A) R(\mu, A) ; \\
= & (\lambda-\mu) R(\lambda, A) R(\mu, A) .
\end{aligned}
$$

Proposition 2.11. Let $A \in B(X)$ be a spectral operator such that $U_{A}=$ $B(0,1)$, let $\left(A^{n}\right)_{n \in \mathbf{N}}$ be a discrete semigroup of contractions on $X$, then for all $\lambda \in C_{A},\|R(\lambda, A)-I\| \leq|\lambda|$.

Proof. Let $A \in B(X)$ be a spectral operator, then for all $\lambda \in C_{A}, R(\lambda, A)=$ $\sum_{n=0}^{\infty} \lambda^{n} A^{n}$. Hence, for all $\lambda \in C_{A}$,

$$
\begin{aligned}
\|R(\lambda, A)-I\| & =\left\|\sum_{n=1}^{\infty} \lambda^{n} A^{n}\right\|, \\
& \leq \sup _{n \geq 1}\left\|\lambda^{n} A^{n}\right\|, \\
& \leq|\lambda| .
\end{aligned}
$$


Proposition 2.12. Let $A \in B(X)$ be a spectral operator such that for all $n \in \mathbf{N},\left\|A^{n}\right\| \leq 1$, then for all $n \in \mathbf{N}, \alpha \in C_{A}^{*}, \lambda \in \Omega_{|\alpha|}$, $R^{(n)}(\lambda, A)=\frac{n !(R(\lambda, A)-I)^{n} R(\lambda, A)}{\lambda^{n}}$.

Proof. Using Proposition 2.10, for all $\lambda, \mu \in \Omega_{|\alpha|}$ whith $\alpha \in C_{A}^{*}$,

$$
(\lambda I+(\mu-\lambda) I+(\lambda-\mu) R(\lambda, A)) R(\mu, A)=\lambda R(\lambda, A) .
$$

Then

$$
\left(I-\frac{1}{\lambda}(\mu-\lambda)(R(\lambda, A)-I)\right) R(\mu, A)=R(\lambda, A) .
$$

The quantity in square brackets on the left of this equation is invertible for $|\lambda|^{-1}|\mu-\lambda|\|R(\lambda, A)-I\|<1$. Thus

$$
R(\mu, A)=\sum_{n=0}^{\infty} \frac{(R(\lambda, A)-I)^{n} R(\lambda, A)}{\lambda^{n}}(\mu-\lambda)^{n} .
$$

But it follows from Proposition 1.6 that $R(\mu, A)$, is analytic on $B(\lambda,|\alpha|)$. From $A \in B(X)$ is spectral operator, then for all $s, t \in \Omega_{|\alpha|}, R(\mu, A)$ can be written as follows:

$\mathrm{R}(\mu, A)=\sum_{n=0}^{\infty} \frac{R^{(n)}(\lambda, A)}{n !}(\mu-\lambda)^{n}$.

Hence, for all $n \in \mathbf{N}, \lambda \in \Omega_{|\alpha|}$,

$\mathrm{R}^{(n)}(\lambda, A)=\frac{n !(R(\lambda, A)-I)^{n} R(\lambda, A)}{\lambda^{n}}$.

We have the following theorem.

Theorem 2.13. Let $X$ be a non-archimedean Banach space over $\mathbf{C}_{p}$, and $A \in B(X)$ be a spectral operator, then for all $n \in \mathbf{N},\left\|A^{n}\right\| \leq 1$ if and only if

$$
\left\|(R(\lambda, A)-I)^{n} R(\lambda, A)\right\| \leq|\lambda|_{p}^{n}
$$

for all $\lambda \in \Omega_{|\alpha|}$ where $\alpha \in C_{A}^{*}$ and $R(\lambda, A)=(I-\lambda A)^{-1}$. 
Proof. Assume that for all $n \in \mathbf{N},\left\|A^{n}\right\| \leq 1$, let $\alpha \in C_{A}^{*}$, by Proposition 1.6, $R(\lambda, A)=(I-\lambda A)^{-1}=\sum_{k=0}^{\infty} \lambda^{k} A^{k}$ is analytic on $\Omega_{|\alpha|}$. Using Proposition 2.12 , for all $n \in \mathbf{N}, \lambda \in \Omega_{|\alpha|}$,

$$
R^{(n)}(\lambda, A)=\frac{n !(R(\lambda, A)-I)^{n} R(\lambda, A)}{\lambda^{n}},
$$

and

$$
\mathrm{R}^{(n)}(\lambda, A)=\sum_{k=n}^{\infty} k(k-1) \cdots(k-n+1) \lambda^{k-n} A^{k}==\sum_{k=n}^{\infty} n !\left(\begin{array}{l}
k \\
n
\end{array}\right) \lambda^{k-n} A^{k},
$$

then for all $n \in \mathbf{N}$ and $\lambda \in \Omega_{|\alpha|}$,

$$
\begin{aligned}
\left\|\frac{R^{(n)}(\lambda, A)}{n !}\right\| & =\left\|\sum_{k=n}^{\infty}\left(\begin{array}{l}
k \\
n
\end{array}\right) \lambda^{k-n} A^{k}\right\|, \\
& \leq \sup _{k \geq n}\left|\left(\begin{array}{l}
k \\
n
\end{array}\right)\right| p|\lambda|_{p}^{k-n}\left\|A^{k}\right\|, \\
& \leq \sup _{k \geq n}|\lambda|_{p}^{k-n}\left\|A^{k}\right\|, \\
& \leq 1 .
\end{aligned}
$$

Thus, for all $n \in \mathbf{N}$ and $t \in \Omega_{|\alpha|}$,

$$
\left\|\frac{R^{(n)}(\lambda, A)}{n !}\right\| \leq 1
$$

From 2.9 and 2.14, we have for all $n \in \mathbf{N}, \lambda \in \Omega_{|\alpha|}$,

$$
\left\|(R(\lambda, A)-I)^{n} R(\lambda, A)\right\| \leq|\lambda|_{p}^{n}
$$

Conversely, let $A \in B(X)$ be a spectral operator, we assume that 2.8, for all $\lambda \in \Omega_{|\alpha|}, R(\lambda, A)=\sum_{n=0}^{\infty} \lambda^{n} A^{n}$. Set for all $\lambda \in \Omega_{|\alpha|}, k \in \mathbf{N}, S_{k}(\lambda)=$ $\lambda^{-k}(R(\lambda, A)-I)^{k} R(\lambda, A)$, then for all $\lambda \in \Omega_{|\alpha|}, k \in \mathbf{N},\left\|S_{k}(\lambda)\right\| \leq 1$. Since $A$ and $R(\lambda, A)$ commute, we have:

$$
\begin{aligned}
S_{k}(\lambda) & =\lambda^{-k}((I-(I-\lambda A)) R(\lambda, A))^{k} R(\lambda, A), \\
& =\lambda^{-k}(\lambda A R(\lambda, A))^{k} R(\lambda, A), \\
& =A^{k} R(\lambda, A)^{k+1} .
\end{aligned}
$$


Then for all $\lambda \in \Omega_{|\alpha|}, k \in \mathbf{N}$,

$$
\begin{aligned}
\left\|A^{k}\right\| & =\left\|(I-\lambda A)^{k+1} S_{k}(\lambda)\right\|, \\
& \leq\left\|(I-\lambda A)^{k+1}\right\|\left\|S_{k}(\lambda)\right\|, \\
& \leq\left\|\sum_{j=0}^{k+1}\left(\begin{array}{c}
k+1 \\
j
\end{array}\right)(-\lambda A)^{j}\right\|, \\
& \leq \max \left\{1,\|\lambda A\|,\left\|\lambda^{2} A^{2}\right\|, \cdots,\left\|\lambda^{k+1} A^{k+1}\right\|\right\}
\end{aligned}
$$

for $\lambda \rightarrow 0$, we have for all $k \in \mathbf{N},\left\|A^{k}\right\| \leq 1$.

For $A$ densely closed linear operator on $X$, the resolvant set $\rho(A)$ is the set of all $\lambda \in \mathbf{K}$ such that the range $\operatorname{Im}(\lambda-A)$ is dense in $X$ and that $\lambda I-A$ has the continous inverse $(\lambda I-A)^{-1}$ on $D\left((\lambda I-A)^{-1}\right)=\operatorname{Im}(\lambda I-A)$, (where $\operatorname{Im}(\lambda I-A)$ denote the range of $(\lambda I-A))$. In the next statements, we assume that $\mathbf{K}$ is a non-archimedean non trivially complete valued field with valuation $|\cdot|$.

Theorem 2.14. Let $X$ be a non-archimedeab Banach space of countable type over $\mathbf{K}$, let $\left(A^{n}\right)_{n \in \mathbf{N}}$ be a discrete semigroup of genrator $A$ be densely defined closed linear operator on $X$ such that $\operatorname{Im}(A) \subset D(A)$. Suppose that $\rho(A) \neq \emptyset$, then $A$ is bounded.

Proof. Let $\left(A^{n}\right)_{n \in \mathbf{N}}$ be a discrete semigroup, suppose that $\rho(A) \neq \emptyset$, let $\lambda \in \rho(A)$, hence $(\lambda I-A)^{-1} \in B(X)$, then there exists $M>0$ such that

$$
\text { for all } x \in D(\lambda I-A)=D(A),\|(\lambda I-A) x\| \geq M\|x\| .
$$

Or $A$ be densely closed linear operator, then $\operatorname{Im}(\lambda I-A)$ is closed. In fact, $x_{n} \in D(A)$ and $z \in X,(\lambda I-A) x_{n} \rightarrow z$. By inequality 2.23, $\left(x_{n}\right)$ is a Chauchy sequence in $X$. Or $X$ is complete, then $x_{n} \rightarrow x$, for some $x \in X$. Thus, $x_{n} \rightarrow x$ and $A x_{n} \rightarrow \lambda x-z$. By the closedness of $A$, we have $x \in D(A)$ and $A x=\lambda x-z$. Since $\lambda \in \rho(A), \operatorname{Im}(\lambda I-A)$ is dense in $X$, then $\operatorname{Im}(\lambda I-A)=X$. Consequently, $X=\operatorname{Im}(\lambda I-A) \subseteq D(A)$, hence $D(A)=X$, then $A$ is bounded.

Proposition 2.15. Let $X$ be a non-archimedean Banach space over $\mathbf{K}$, let $A \in B(X)$ such that $\|A\|<1$. Let $q(\lambda)$ be an arbitrary polynomial and set $p(\lambda)=1-(1-\lambda) q(\lambda)$. Then we have

$$
\|p(A)\|=\left\|(I-A)^{-1}-q(A)\right\| \text {. }
$$


Proof. Let $A \in B(X)$ such that $\|A\|<1$. Let $q(\lambda)$ be an arbitrary polynomial and set $p(\lambda)=1-(1-\lambda) q(\lambda)$, then

$$
\begin{aligned}
(I-A)^{-1}-q(A) & =(I-A)^{-1}(I-(I-A) q(A)) \\
& =(I-A)^{-1} p(A) .
\end{aligned}
$$

Thus,

$$
\begin{aligned}
\left\|(I-A)^{-1}-q(A)\right\| & =\left\|(I-A)^{-1} p(A)\right\|, \\
& \leq\|p(A)\| .
\end{aligned}
$$

On the other hand,

$$
p(A)=(I-A)\left((I-A)^{-1}-q(A)\right) .
$$

Hence,

$$
\begin{aligned}
\|p(A)\| & =\left\|(I-A)\left((I-A)^{-1}-q(A)\right)\right\|, \\
& \leq\|(I-A)\|\left\|\left((I-A)^{-1}-q(A)\right)\right\|, \\
& \leq\left\|\left((I-A)^{-1}-q(A)\right)\right\| .
\end{aligned}
$$

Then,

$$
\|p(A)\|=\left\|(I-A)^{-1}-q(A)\right\| .
$$




\section{References}

[1] T. Diagana, Non-archimedean linear operators and applications. Huntington, NY: Nova Science, 2007.

[2] A. El Amrani, A. Blali, J. Ettayb, and M. Babahmed, "A note on C0-groups and C-groups on non-archimedean Banach spaces", Asian-European journal of mathematics, vol. 14, no. 06, Art. ID. 2150104, 2020.

[3] A. G. Gibson, "A discrete Hille-Yoshida-Phillips theorem", Journal of mathematical analysis and applications, vol. 39, pp. 761-770, 1972.

[4] N. Koblitz, P-adic analysis: A short course on recent work. Cambridge: Cambridge University Press, 1980.

[5] A. C. M. van Rooij, Non-Archimedean functional analysis. New York, NY: Dekker, 1978.

[6] W. H. Schikhof, "On p-adic compact operators", Catholic University, Department of Mathematics, Nijmegen, The Netherlands, Tech. Rep. 8911, 1989.

[7] A. Pazy, Semigroups of linear operators and applications to partial differential equations. New York, NY: Springer, 1983. 


\author{
Abdelkhalek El amrani \\ Department of mathematics and computer science, \\ Sidi Mohamed Ben Abdellah University, \\ Faculty of Sciences Dhar El Mahraz, \\ Fès, \\ Morocco \\ e-mail: abdelkhalek.elamrani@usmba.ac.ma

\section{Jawad Ettayb} \\ Department of mathematics and computer science, \\ Sidi Mohamed Ben Abdellah University, \\ Faculty of Sciences Dhar El Mahraz, \\ Fès, \\ Morocco \\ e-mail: jawad.ettayb@usmba.ac.ma \\ Corresponding author \\ and
}

\author{
Aziz Blali \\ Department of Mathematics, \\ Sidi Mohamed Ben Abdellah University, \\ ENS \\ B. P. 5206 Bensouda-Fès, \\ Morocco \\ e-mail: aziz.blali@usmba.ac.ma
}

